\title{
Acumulación de selenio en setas silvestres comestibles: captación y toxicidad Selenium accumulation in wild edible mushrooms: uptake and toxicity
}

\section{J. Melgar , J. Alonso \& M. A. García}

To cite this article: M. J. Melgar , J. Alonso \& M. A. García (2009) Acumulación de selenio en setas silvestres comestibles: captación y toxicidad Selenium accumulation in wild edible mushrooms: uptake and toxicity, CyTA - Journal of Food, 7:3, 217-223, DOI: 10.1080/19476330903068688

To link to this article: https://doi.org/10.1080/19476330903068688

\section{(2) Copyright Taylor and Francis Group, LLC}

曲 Published online: 08 Mar 2010.

Submit your article to this journal

Llll Article views: 759

Q View related articles $\asymp$

Citing articles: 4 View citing articles $\longleftarrow$ 


\title{
Acumulación de selenio en setas silvestres comestibles: captación y toxicidad Selenium accumulation in wild edible mushrooms: uptake and toxicity
}

\author{
M.J. Melgar*, J. Alonso and M.A. García \\ Área de Toxicología, Facultades de Veterinaria y Ciencias, Universidad de Santiago de Compostela (Campus de Lugo) C/ Carvalho \\ Calero $s / n, 27002-L U G O$, España
}

(Received 26 November 2008; final version received 27 May 2009)

\begin{abstract}
Selenium is considered as a trace element metal, but in excessive doses it becomes toxic for the consumer's health. In this study, the goal was to determine the accumulation capacity of selenium in edible mushrooms in relation to some factors and their possible toxicological implications. Edible mushrooms (97 samples of 14 species) were collected in Lugo, Galicia (Spain). Anatomical parts, hymenophore and the rest of the fruiting body, were processed by digestion in acid medium in a microwave oven and analyzed by inductively coupled plasma-optical emission spectrometer. Boletus pinicola and Boletus edulis reached the highest mean selenium contents in hymenophore with values of 74.93 and $52.7 \mathrm{mg} / \mathrm{kg}$ dry weight, respectively. Organic matter in growth soil between 12-16\% favoured its uptake. Finally, taking into account the dose of chronic toxicity of selenium, relatively close to the dietary recommendations (WHO), and the concentrations obtained in the studied mushrooms, a moderate consumption of some species of Boletus is
\end{abstract} recommended.

Keywords: selenium; edible mushrooms; ICP-OES

Los hongos acumulan selenio, metal considerado nutricionalmente oligoelemento, pero nocivo para la salud del consumidor en dosis excesivas. Se estudió el contenido de selenio en setas silvestres comestibles, teniendo en cuenta los factores influyentes en su captación, y el riesgo toxicológico derivado de su consumo. 97 muestras de setas de 14 especies fueron recogidas en Lugo, Galicia (España). Separadamente, las porciones anatómicas: himenóforo y resto del carpóforo, fueron digeridas en medio ácido en un microondas, y analizadas mediante la técnica de inducción de plasma acoplado a espectrometría de emisión óptica. Boletus pinicola y Boletus edulis son las especies más acumuladoras de selenio en himenóforo con 74,93 y $52,7 \mathrm{mg} / \mathrm{kg}$ p.s., respectivamente. La materia orgánica del suelo entre $12-16 \%$ favoreció su absorción. Finalmente, considerando la dosis de toxicidad crónica del selenio, relativamente próxima a las recomendaciones dietéticas (OMS), y las concentraciones de las setas estudiadas, se aconseja el consumo moderado de algunas especies del género Boletus.

Palabras clave: selenio; setas comestibles; ICP-OES

\section{Introducción}

La polución metálica medioambiental ha incrementado notablemente el interés por el estudio de los hongos ya que son captadores de metales pesados. El estudio de las interacciones entre metales pesados y hongos está motivado, entre otras cosas, por la ubicuidad y presencia de los hongos en hábitat contaminados, así como por la captación y traslocación de metales pesados a los carpóforos (setas) de macromicetos comestibles, por sus repercusiones sanitarias y ecotoxicológicas, incluso por sus posibles aplicaciones biotecnológicas derivadas de la capacidad de algunos hongos para captar y fijar metales (Quinche, 1987; Gadd, 1993; Melgar, Alonso, Pérez-López, \& García, 1998; Alonso, Salgado, García, \& Melgar, 2000; Zaichick, 2002; Alonso, García, Pérez-López, \& Melgar, 2003, 2004; Jonnalagadda, Pienaar, \& Haripersad, 2006; Yamac,
Yildiz, Sarikurkcu, Celikkollu, \& Solak, 2007; Falandysz et al., 2008a, 2008b).

Existen factores que influyen en la disponibilidad de los metales pesados del suelo, siendo particularmente importantes en el estudio del impacto de la contaminación del suelo para los organismos que se nutren de él, especialmente plantas y hongos, e indirectamente para la salud animal y humana por el consumo de éstos. Entre estos factores se encuentran el $\mathrm{pH}$ y la materia orgánica del suelo, así como las formas químicas del elemento y sus interacciones (Alloway, 1995).

Nutricionalmente, el selenio se considera un oligoelemento, que actúa fundamentalmente como antioxidante al formar parte de la enzima glutatión peroxidasa que le confiere una función protectora frente al cáncer y enfermedades cardiovasculares y un efecto inhibidor de la formación de radicales libres 
retrasando el proceso de envejecimiento (Hernández Rodríguez, \& Sastre Gallego, 1999). En general, el selenio es necesario en la biosíntesis de selenoenzimas y selenoproteínas importantes, tales como yodotironina 5'-deyodasas, tioredoxín reductasas, selenoproteína P y selenoproteína $\mathrm{W}$, las cuales intervienen en diversas vías metabólicas y expresión génica y contribuyen al cuidado de la alimentación y contribuyen a la salud humana (Combs, 2001; Whanger, 2002, 2004; Dumont, Vanhaecke, \& Cornelis, 2006; Burk \& Hill, 2005). Las ingestas diarias recomendadas por la OMS (2003) son: $1,7 \mu \mathrm{g} / \mathrm{kg}$ p.c. para niños y $0,9 \mu \mathrm{g} / \mathrm{kg}$ p.c. para adultos. Incluso se ha llegado a establecer que la toxicidad de metales como el mercurio y cadmio en experimentación animal ha sido reducida si la dieta contenía, también, niveles adecuados de selenio (Stijve, 1977).

Sin embargo, en dosis excesivas, superiores a $30 \mu \mathrm{g} / \mathrm{kg}$ p.c. (OMS, 2003), el selenio es nocivo para la salud, destacando los problemas relacionados con pérdida de dientes, del cabello y de las uñas (Shils, Olson, Shike, \& Ross, 2002). Además, paradójicamente, Letavayová, Vlčkvá, V. and Brozmanová (2006) consideraron que el selenio en grandes dosis puede ser citotóxico y hasta un agente carcinógeno.

En general, el contenido de selenio en setas varía mucho de unas especies a otras, si bien algunos géneros, tales como Albatrellus, Amanita, Boletus, Agaricus y Macrolepiota, son conocidos por su gran capacidad de acumulación, siendo los tubos y las laminillas, generalmente, las partes morfológicas del carpóforo en las que más abunda el selenio frente al estipe o pie (Stijve, 1977; Piepponen, Liukkonen-Lilja, \& Kuusi, 1983; Jorhem \& Sundström, 1995; Slejkovec et al., 2000; Falandysz, 2003, 2008; Falandysz \& Lipka, 2006; Falandysz et al., 2008); no obstante, las especies cultivadas (Agaricus bisporus) contienen bastante menos selenio que incluso frutas y vegetales (Stijve, 1977).

Por todo ello, los objetivos de este trabajo son la determinación del contenido de selenio total en muestras de setas comestibles silvestres, el análisis estadístico de los factores que influyen en su captación, y finalmente la evaluación del riesgo toxicológico que se derive de su consumo.

\section{Material y métodos}

\section{Recolección de muestras}

En este trabajo se recogieron 97 muestras de setas comestibles no cultivadas pertenecientes a 14 especies de la clase Basidiomycetes, siete saprófitas y siete micorrízicas (factor ecología): Boletus edulis, Boletus pinicola, Boletus badius, Lactarius deliciosus, Russula cyanoxantha, Tricholoma portentosum, Agrocybe aegerita, Agaricus campestris, Agaricus macrosporus, Coprinus comatus, Macrolepiota procera, Fistulina hepatica, Hydnum repandum y Lepista nuda. Las especies se seleccionaron en base a su índice de consumo, comercialización y frecuencia en las zonas de muestreo.
Se diferenciaron dos zonas de recogida en la provincia de Lugo, Galicia (España), en razón de la mayor o menor contaminación antropogénica: (1) Zonas urbanas y anexas a carreteras $(\mathrm{pH}=7)$; y (2) zonas libres de contaminación aparente (campestres $\mathrm{pH}=5$ o forestales $\mathrm{pH}=4$ ) alejadas de núcleos urbanos, carreteras u otras fuentes de contaminación.

\section{Preparación de las muestras}

Las muestras se prepararon según el método utilizado en otros estudios (García, Alonso, Fernández, \& Melgar, 1998): los carpóforos se limpiaron y se separaron dos partes anatómicas (himenóforo o zona fértil en donde se forman las esporas sexuales, y el resto del carpóforo). Posteriormente se procedió a la homogeneización en un homogeneizador de vidrio borosilicado con émbolo de pistón en PTFE estriado. El homogeneizado de cada porción fue transferido a frascos estériles de polietileno, la conservación se realizó mediante congelación. El procedimiento de digestión se llevó a cabo en medio ácido (una mezcla de ácido nítrico y clorhídrico en una proporción 3:2 y diluidos al 50\% en agua milli-Q) en una estación de microondas de laboratorio (ETHOS 20, Milestone), equipado con vasos cerrados de teflón TFM y sometiendo todo ello a elevadas presiones, y bajo control de temperatura.

Paralelamente, se preparó una muestra de cada porción (1,5-3 g) que sería pesada en crisol de porcelana para su posterior desecación en estufa $\left(110^{\circ} \mathrm{C}\right)$, hasta obtener peso constante, y así poder determinar el peso seco de las muestras, al cual se refieren los resultados.

\section{Técnicas analiticas}

Una vez digeridas las muestras, los metales en disolución se determinaron mediante la técnica de inducción de plasma acoplado a espectrometría de emisión óptica, utilizando un ICP-OES Perkin Elmer OPTIMA 4300 DV, y el programa WinLab32. La longitud de onda fue: $196,027 \mathrm{~nm}$. El tiempo de integración fue variable entre $1-5 \mathrm{~s}$, con tres réplicas por muestra, muestreador automático y lavado con ácido nítrico al 2\%. El caudal del argón fue regulado a $15 \mathrm{ml} / \mathrm{min}$, el flujo de muestra fue de $1,5 \mathrm{ml} / \mathrm{min}$ y el potencial de $1300 \mathrm{~W}$.

La calidad del método se valoró mediante el estudio de los parámetros de sensibilidad (límite de detección), exactitud (material de referencia del liquen Evernia prunastri L. y recuperaciones analíticas) y precisión (coeficiente de variación).

Para determinar el $\mathrm{pH}$, se tomaron $10 \mathrm{~g}$ de muestra de suelo y se le añadieron $25 \mathrm{ml}$ de agua destilada y la mezcla se dejó a $25^{\circ} \mathrm{C}$ durante $1 \mathrm{~h}$. A continuación se midió el $\mathrm{pH}$ usando un medidor de pH (GLP 21, GRISON).

El contenido de materia orgánica fue determinado gravimétricamente después de someter $2 \mathrm{~g}$ de muestra a 
$550{ }^{\circ} \mathrm{C}$ durante $16 \mathrm{~h}$ en un horno mufla (Select-Horn, SELECTA) (Falandysz \& Bielawski, 2007).

\section{Análisis estadístico}

Los datos obtenidos fueron tratados estadísticamente mediante el programa SPSS 13.0 (SPSS Inc., Illinois, USA) con un diseño experimental completamente aleatorizado y siguiendo el procedimiento de t pareado y univariante considerando las diversas variables: especie, parte anatómica, tráfico, hábitat, $\mathrm{pH}$ y materia orgánica del suelo.

\section{Resultados y discusión}

De acuerdo con los resultados obtenidos en este trabajo, la concentración media de selenio en las especies estudiadas, según las porciones anatómicas, fue de $18,22 \mathrm{mg} / \mathrm{kg}$ y $11,92 \mathrm{mg} / \mathrm{kg}$ para himenóforo y resto del carpóforo, respectivamente. Todos los resultados presentados están referidos a peso seco (p.s.).

La especie se ha mostrado como un factor esencial en cuanto a la acumulación de selenio (Tabla 1), tal como refieren algunos investigadores (Stijve, 1977; Piepponen et al., 1983; Falandysz, 2008), destacando como grandes acumuladoras de selenio, Boletus pinicola y Boletus edulis tanto en himenóforo (74,93 y $52,73 \mathrm{mg} / \mathrm{kg}$, respectivamente) como en el resto del carpóforo (33,94 y 30,19 mg/kg, respectivamente). Ambas especies difieren significativamente su concentración de selenio en el himenóforo con el resto de las especies. Sin embargo, en el resto del carpóforo, no se aprecian diferencias estadísticamente significativas entre las medias poblacionales de extracción para Boletus pinicola y el resto de las especies, mientras que sí existen entre Boletus edulis y siete de las demás especies. Se observaron diferencias estadísticamente significativas según la parte anatómica (himenóforo y resto del carpóforo) en Agaricus macrosporus $(\mathrm{p}<0,01)$, Boletus edulis $(\mathrm{p}<0,001)$ y Macrolepiota procera $(\mathrm{p}<0,05)$. Por contraste, entre las especies estudiadas, Hydnum repamdum y Lactarius deliciosus, fueron las menos acumuladoras de selenio en ambas porciones: himenóforo 3,65 y $3,40 \mathrm{mg} / \mathrm{kg}$ respectivamente; y resto del carpóforo en torno a $3 \mathrm{mg} / \mathrm{kg}$.

Según la revisión bibliográfica realizada por Falandysz (2008) sobre el contenido de selenio en hongos comestibles, cuando considera las partes anatómicas donde el selenio se acumula, menciona, preferentemente, el himenóforo o sombrerillo (tubos/ laminillas y parte carnosa) versus el estipe o pie (resto del carpóforo). Además, muestra que el contenido de selenio en setas es relativamente pequeño y muy dispar según la especie considerada. Destaca que la especie Albatrellus pes-caprae (no considerada en este trabajo), con hasta un promedio de $200 \mu \mathrm{g} \mathrm{Se} / \mathrm{g}$ p.s., es la más acumuladora; seguida del género Boletus, concretamente Boletus pinicola y Boletus edulis con un promedio de 40 y $20 \mu \mathrm{g} \mathrm{Se} / \mathrm{g}$ p.s., respectivamente. Estos datos son similares a los obtenidos en las especies estudiadas en este trabajo; y asimismo, coincide en que algunas especies de los géneros Agaricus silvestres, tales como A. campestris y $A$. macrosporus $(2-5 \mu \mathrm{g} \mathrm{Se} / \mathrm{g}$ p.s.), entre otras, también destacaron por ser relativamente ricas en selenio. Piepponen et al. (1983) y Falandysz (2003) señalaron la especie Macrolepiota procera por su considerable contenido en selenio $(5 \mathrm{mg} / \mathrm{kg})$, al mismo tiempo que reconocían al género Lactarius, junto con otras especies, por su bajo contenido en selenio, tal y como hemos mostrado en nuestras observaciones.

En el estudio estadístico, los diagramas de caja están considerados como los más explicativos ya que además de mostrar la mediana de la distribución,

Tabla 1. Valores medios ( $\mathrm{mg} / \mathrm{kg}$ p.s.) y desviación estándar del contenido en selenio según especie y parte anatómica (diferencias estadísticamente significativas: $* \mathrm{p}<0,05 ; * * \mathrm{p}<0,01 ; * * \mathrm{p}<0,001)$.

Table 1. Mean values (mg/kg d.w.) and standard deviations of selenium contents according to species and anatomical parts (significant differences: $* \mathrm{p}<0.05 ; * * \mathrm{p}<0.01 ; * * \mathrm{p}<0.001$ ).

\begin{tabular}{|c|c|c|}
\hline Especie & $\mathrm{Se}-\mathrm{h}^{1}$ & Se-rc ${ }^{2}$ \\
\hline Agaricus camprestris $(\mathrm{n}=7)$ & $19,38 \pm 17,03$ & $11,30 \pm 0,70$ \\
\hline Agaricus macrosporus $(\mathrm{n}=11)$ & $7,98 * * \pm 2,84$ & $13,35 * * \pm 5,94$ \\
\hline Agrocybe aegerita $(\mathrm{n}=3)$ & $9,55 \pm 7,72$ & $5,00 \pm 1,96$ \\
\hline Boletus badius $(\mathrm{n}=8)$ & $6,91 \pm 3,60$ & $9,43 \pm 5,27$ \\
\hline Boletus edulis $(\mathrm{n}=10)$ & $52,73 * * * \pm 22,77$ & $30,19 * * * \pm 15,25$ \\
\hline Boletus pinicola $(\mathrm{n}=8)$ & $74,93 \pm 63,51$ & $\mathbf{3 3 , 9 4 \pm 2 2 , 6 5}$ \\
\hline Coprinus comatus $(\mathrm{n}=5)$ & $4,33 \pm 3,18$ & $4,03 \pm 3,73$ \\
\hline Fistulina hepatica $(\mathrm{n}=3)$ & $5,70 \pm 3,07$ & $1,96 \pm 3,39$ \\
\hline Hydnum repandum $(\mathrm{n}=7)$ & $3,65 \pm 2,84$ & $3,14 \pm 5,57$ \\
\hline Lactarius deliciosus $(\mathrm{n}=7)$ & $3,40 \pm 3,65$ & $3,16 \pm 3,81$ \\
\hline Lepista nuda $(\mathrm{n}=7)$ & $3,96 \pm 2,63$ & $10,74 \pm 8,30$ \\
\hline Macrolepiota procera $(\mathrm{n}=6)$ & $16,16^{*} \pm 8,66$ & $5,52 * \pm 2,35$ \\
\hline Russula cyanoxantha $(\mathrm{n}=7)$ & $6,47 \pm 3,95$ & $6,88 \pm 4,14$ \\
\hline Tricholoma portentosum $(\mathrm{n}=8)$ & $9,37 \pm 7,91$ & $4,92 \pm 6,03$ \\
\hline
\end{tabular}

1: Himenóforo; 2: resto del carpóforo.

1: Hymenophore; 2: rest of the fruiting body. 
también muestran la variabilidad al representar las bisagras de Tukey y los valores más alejados de los centrales; con un círculo se señalan los que se alejan más de 1,5 veces la longitud de caja de la bisagra de Tukey, y con asterisco los que doblan esa desviación. Adicionalmente, se añade la numeración ordinal del dato para verificar la posibilidad de un dato anómalo. Así, en este trabajo, los diagramas (Figuras 1 y 2) son muy explicativos de los resultados que dan los contrastes con Boletus pinicola y Boletus edulis, los cuales presentan concentración media y desviación típica muy superiores a los de las demás especies. Puede

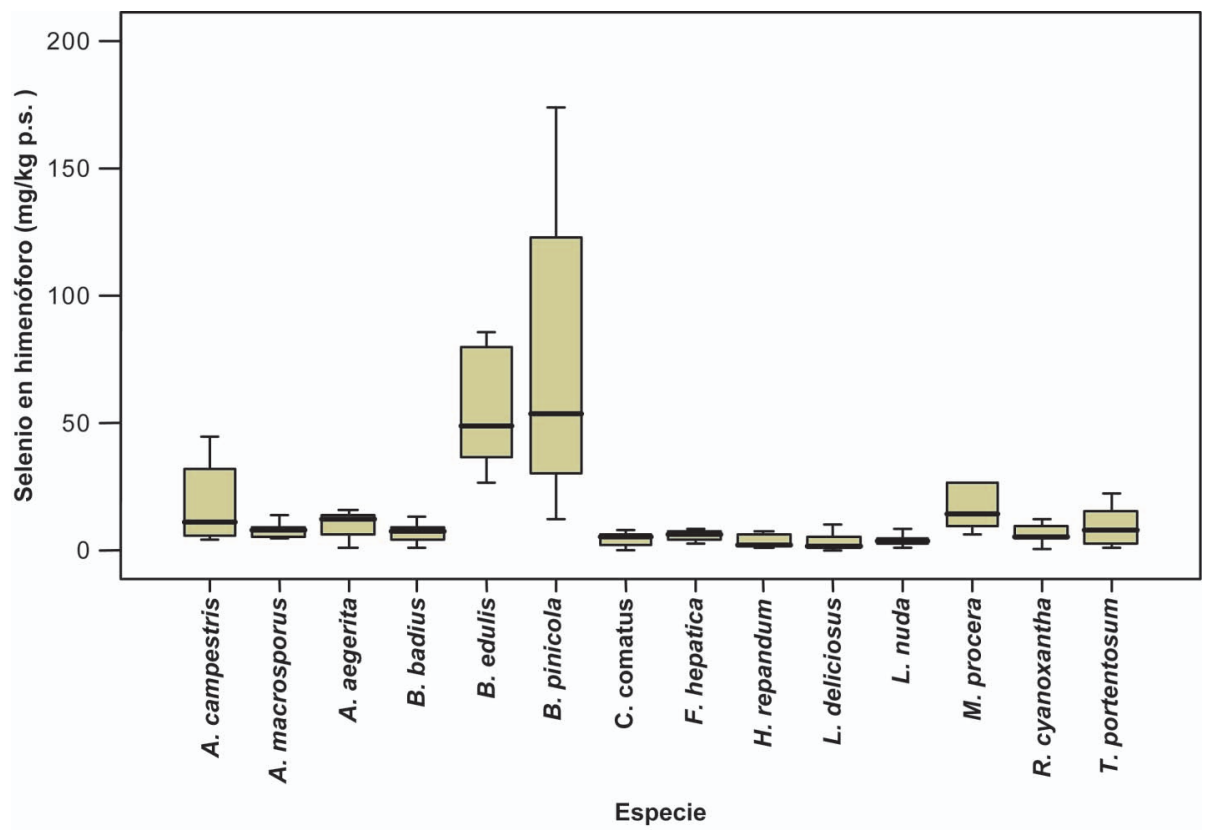

Figura 1. Variabilidad del contenido de selenio (mg/kg p.s.) en himenóforo según la especie.

Figure 1. Variability of selenium content (mg/kg d.w.) in hymenophore according to species.

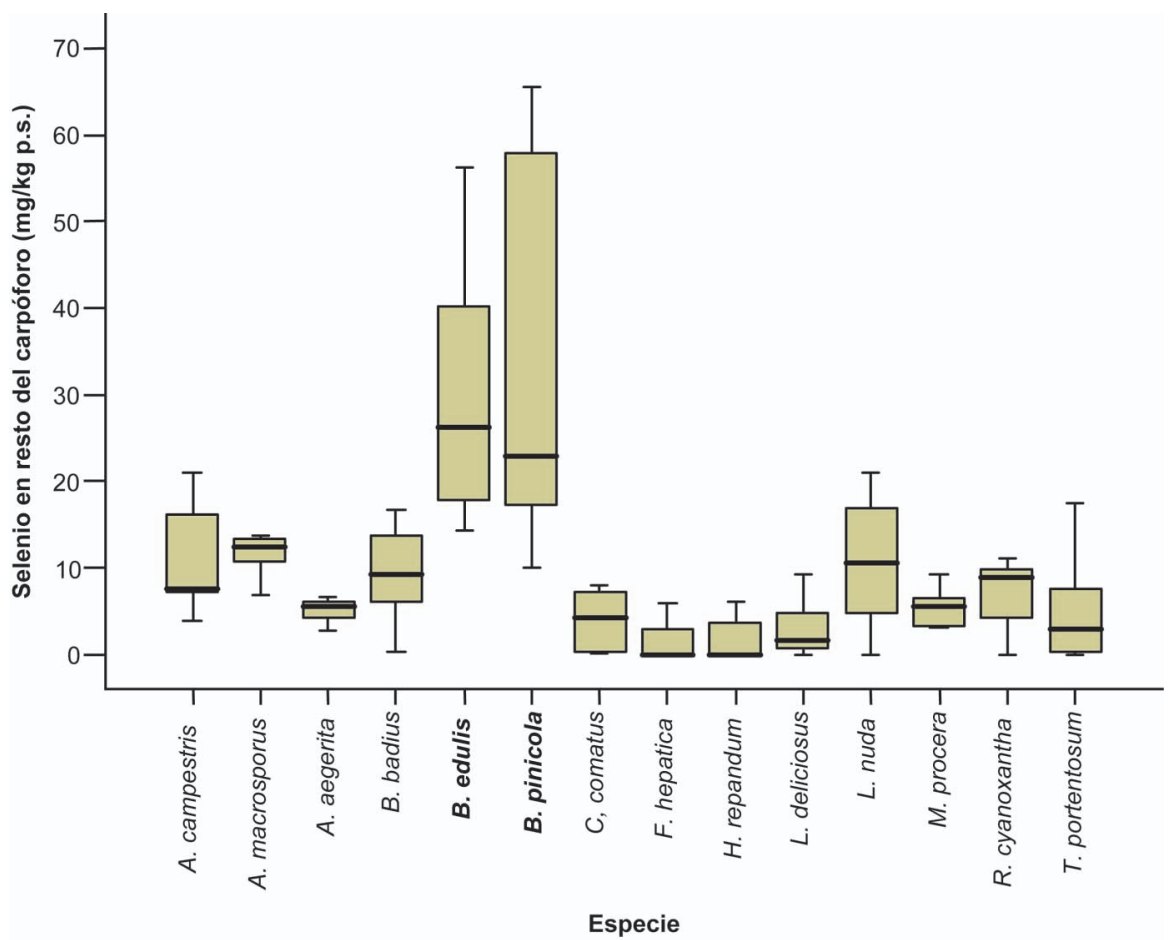

Figura 2. Variabilidad del contenido de selenio ( $\mathrm{mg} / \mathrm{kg}$ p.s.) en resto del carpóforo según la especie.

Figure 2. Variability of the selenium content ( $\mathrm{mg} / \mathrm{kg} \mathrm{d}$.w.) in rest of the fruiting body according to species. 
observarse la aparente igualdad de la concentración media de selenio en el himenóforo del resto de las especies y la inexistencia de presuntos valores anómalos.

De forma significativa, en la acumulación de selenio, también influyó el contenido de materia orgánica del suelo de procedencia de las especies muestreadas, observándose una mayor capacidad cuando éste tenía entre $12-16 \%$ de materia orgánica que se corresponde con el nivel tres del factor en ambas partes anatómicas (Figuras 3(a) y 3(b)).

Respecto al factor $\mathrm{pH}$, es muy indicativo que tanto en el himenóforo como en el resto del carpóforo se aprecie una similitud de medias y una discrepancia clara entre las varianzas, llevándonos a la conclusión de que el pH del suelo no influye en la acumulación de selenio. Sin, embargo, respecto al factor tráfico (contaminación antropogénica), de forma inesperada se observó que la concentración media de selenio en setas fue más elevada en zonas de menor contaminación automovilística, pero no existen diferencias estadísticamente significativas. En la literatura consultada (Demirbas, 2000, 2001; Isildak, Turkekul, Elmastas, \& Tuzen, 2004) no se ha encontrado ninguna referencia a esta situación, pero parece bastante improbable que con la gran variedad de muestras recogidas, coincidiera que las especies obtenidas en suelos con elevado nivel de tráfico fuesen a proceder de
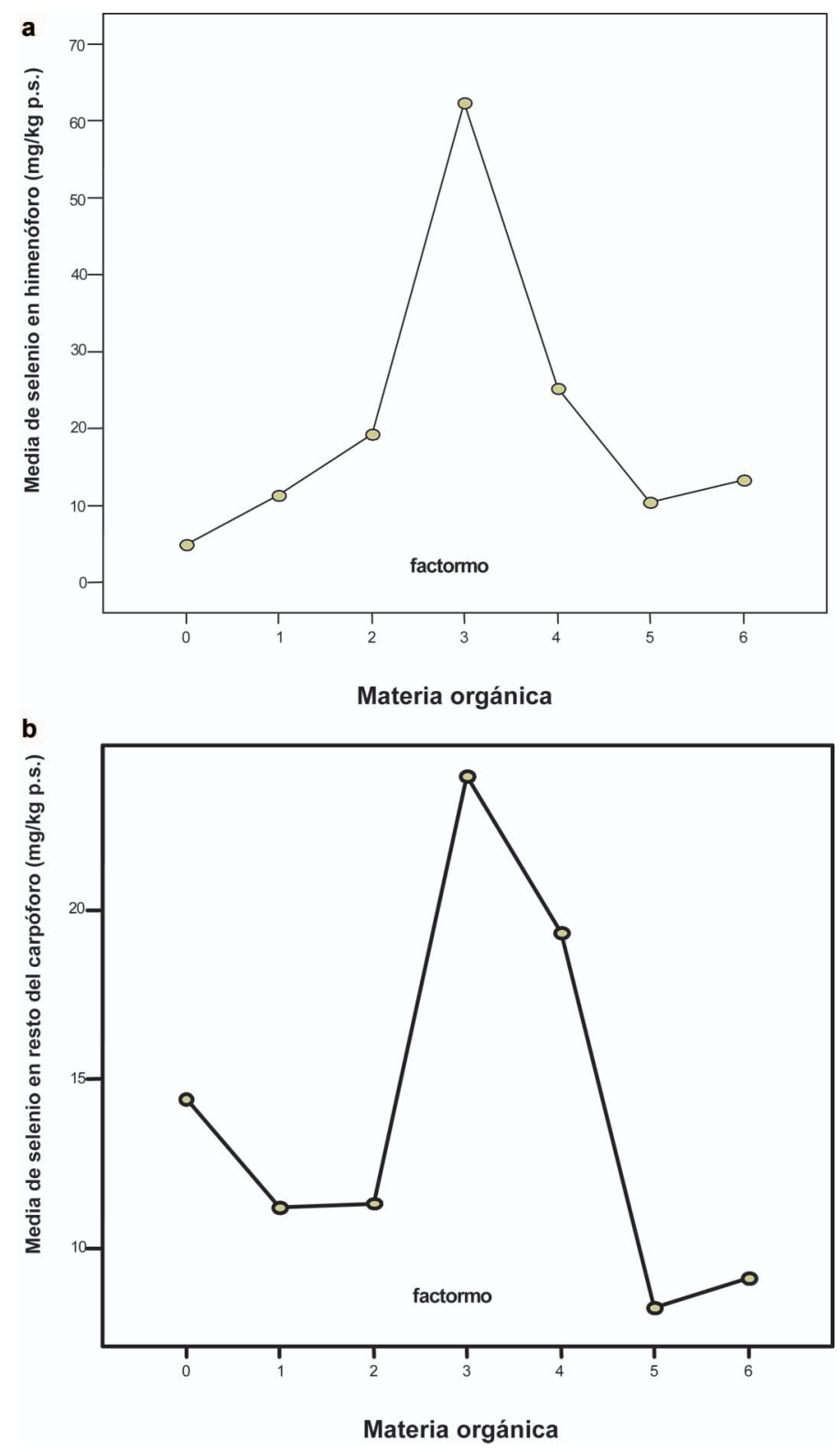

Figure 3. Variabilidad del contenido de selenio en (a) Himenóforo; y (b) resto del carpóforo, frente al factor materia orgánica (factormo).

Figure 3. Variability of selenium content in: (a) Hymenophore; and (b) rest of the fruiting body, according to the organic matter factor (factormo). 
suelos con poco selenio, y las que procedían de suelos no afectados por el tráfico procediesen de suelos con alto contenido en selenio. Para corroborar estos resultados, habría que profundizar estudiando el contenido de selenio en el suelo de procedencia de las muestras.

Considerando el hábitat como factor, la procedencia forestal o de herbazal no afectó a la captación de selenio objeto de estudio. Igual razonamiento puede hacerse acerca de la influencia del factor ecología: carácter de micorriza o saprófita, no existen diferencias estadísticamente significativas en contraste con los resultados obtenidos sobre otros metales (Alonso, Melgar, \& García, 1996; Alonso, Salgado, García, \& Melgar 2000; Alonso, García, Pérez-López, \& Melgar 2003, 2004; García et al., 1998; Melgar, Alonso, PérezLópez, \& García, 1998).

La normativa de aplicación en España, en relación al contenido de metales y otros contaminantes en hongos silvestres o cultivados, es el Reglamento No. 1881/2006 y sus modificaciones (DOUE, 2006, 2008). Sin embargo, en el mismo, no se hace ninguna referencia al selenio. El Real Decreto 30/2009 por el que se establecen las condiciones sanitarias para la comercialización de setas para uso alimentario (BOE, 2009), no contemplan ninguna disposición relativa al contenido de elementos metálicos en estos alimentos, aunque indica, entre los requisitos de las setas comercializadas, el hallarse sin residuos de contaminantes químicos por encima de los límites legalmente establecidos. Sin embargo, desde el punto de vista toxicológico, conocidas las concentraciones medias del selenio en las setas estudiadas (himenóforo: $18,22 \mathrm{mg} / \mathrm{kg}$ y resto del carpóforo: $11,92 \mathrm{mg} / \mathrm{kg}$ ), se pretende discutir el consumo de las mismas para saber si es aceptable. Es de destacar que la toxicidad crónica del selenio $(1950 \mu \mathrm{g} /$ día $)$ está relativamente próxima a las recomendaciones dietéticas (OMS, 2003). Por otra parte, un consumo de 100 gramos semanales de himenóforo de las catorce especies de setas estudiadas aportaría aproximadamente $39 \mu \mathrm{g}$ de selenio; si se consumiera exclusivamente himenóforo de Boletus pinicola supondría, aproximadamente, $113 \mu \mathrm{g}$ diarios de ingesta de selenio, lo cual aportaría entre 0,65 y 1,90 veces la recomendación dietética de consumo de selenio. El consumo de la misma cantidad semanal del resto del carpóforo de setas (Agudo et al., 1999) supondría multiplicar la cantidad diaria recomendada (CDR) por una cantidad de entre 0,43 y 1,24 veces, para el conjunto de las especies estudiadas o para el Boletus pinicola, respectivamente.

Aunque la intoxicación aguda por selenio no es frecuente en el hombre, la acumulación en el organismo de altas cantidades puede dar lugar a una intoxicación crónica cuyos efectos ya fueron descritos. Según la normativa europea más reciente, para metales en alimentos, no hay limitación expresa de contenido para el selenio (DOUE, 2006, 2008).
Aportes tan importantes de minerales como el selenio que, además de su esencialidad, pueden ser tóxicos, deberían estar regulados, o al menos deberían establecerse a nivel nacional y comunitario, recomendaciones de limitación de consumo. De hecho en Alemania, se recomienda no superar el consumo de 200 gramos semanales de setas no cultivadas, en España no existe ninguna recomendación de un organismo oficial al respecto.

Por último, teniendo en cuenta la participación de las setas en la dieta española, gallega y luguesa según el Instituto Nacional de Estadística (INE, 2000), puede considerarse que su aporte de selenio, no supone ningún riesgo para la salud del consumidor, si bien se recomienda el consumo moderado de algunas especies del género Boletus.

\section{Agradecimientos}

A la Xunta de Galicia que subvencionó este trabajo a través del Proyecto INCITE08PXB261087PR.

\section{Referencias}

Agudo, A., Amiano, P., Barcos, A., Barricarte, A., Beguiristain, J.M., Chirlaque, M.D., et al. (1999). Dietary intake of vegetables and fruits among adults in five regions of Spain. Europe Journal of Clinical Nutrition, 53, 174-180.

Alonso, J., Melgar, M.J., \& García, M.A. (1996). La contaminación por plomo y cadmio en hongos comestibles en la provincia de Lugo. Belarra, 13, 43-58.

Alonso, J., Salgado, M.J., García, M.A., \& Melgar, M.J. (2000). Accumulation of mercury in edible macrofungi: Influence of some factors. Archives of Environmental Contamination and Toxicology, 38, 158-162.

Alonso, J., García, M.A., Pérez-López, M., \& Melgar, M.J. (2003). The concentrations and bioconcentration factors of copper and zinc in edible mushrooms. Archives of Environmental Contamination and Toxicology, 44, 180-188.

Alonso, J., García, M.A., Pérez-López, M., \& Melgar, M.J. (2004). Acumulación de metales pesados en macromicetos comestibles y factores que influyen su captación. Revista de Toxicología, 21, 11-15.

Alloway, B.J. (1995). Heavy metals in soils (2a ed.). Glasgow: Blackie and Son Ltd.

BOE. (2009). RD 30/2009 de 16 de enero por el que se establecen las condiciones sanitarias para la comercialización de setas para uso alimentario. Madrid: B.O.E.

Burk, R.F., \& Hill, K.E. (2005). Selenoprotein P. An extracellular protein with unique physical characteristics and a role in selenium homeostasis. Annual Review in Nutrition, 25, 215-235.

Combs, G.F. (2001). Selenium in global food systems. British Journal of Nutrition, 85, 517-547.

Demirbas, A. (2000). Accumulation of heavy metals in some edible mushrooms of Turkey. Food Chemistry, 68, 415419.

Demirbas, A. (2001). Concentrations of 21 metals in 18 species of mushrooms growing in the East Black sea region. Food Chemistry, 75, 453-457.

DOUE (2006). Commission Regulation (EC) No 1881/2006 of 19 December 2006 setting maximum levels for certain contaminants in foodstuffs. DO L 364/8.

DOUE (2008). Commission Regulation (EC) No 629/2008 of 2 July 2008 amending Regulation (EC) No 1881/2006 setting maximum levels for certain contaminants in foodstuffs. DO L 173/6. 
Dumont, E., Vanhaecke, F., \& Cornelis, R. (2006). Selenium speciation from food source to metabolites: A critical review. Analytical and Bioanalytical Chemistry, 385, 1304-1323.

Falandysz, J. (2003). Selenium in selected species of mushrooms from Poland. Roczniki Panstwowego Zakladu Higieny, 54(3), 249-254.

Falandysz, J. (2008). Selenium in edible mushrooms. Journal of Environmental Science and Health, Part C, 26(3), 256-299.

Falandysz, J., \& Lipka, K. (2006). Selenium in mushrooms. Roczniki Panstwowego Zakladu Higieny, 57(3), 217-233.

Falandysz, J., \& Bielawski, L. (2007). Mercury and its bioconcentration factors in Brown Birch Scaber Stalk (Leccinum scabrum) from various sites in Poland. Food Chemistry, 105, 635-640.

Falandysz, J., Kunito, T., Kubota, R., Gucia, M., Mazur, A., Falandysz, J.J., et al. (2008a). Some mineral constituents of Parasol Mushroom (Macrolepiota procera). Journal of Environmental Science and Health, Part $B, 43,187-192$.

Falandysz, J., Kunito, T., Kubota, R., Bielawski, L., Frankowska, A., Falandysz, J.J., et al. (2008b). Multivariate characterization of elements accumulated in King Bolete Boletus edulis mushroom at lowland and high mountain regions. Journal of Environmental Science and Health, Part A, 43(14), 1692-1699.

Gadd, G.M. (1993). Interactions of fungi with toxic metals. New Phytology, 124, 25-60.

García, M.A., Alonso, J., Fernández, M.I., \& Melgar, M.J. (1998). Lead content in edible wild mushrooms in northwest Spain as indicator of environmental contamination. Archives of Environmental Contamination and Toxicology, 34, 330-335.

Hernández Rodríguez M. \& Sastre Gallego A. (1999). Tratado de nutrición. Madrid: Díaz de Santos.

INE (2000). Encuesta de presupuestos familiares 1991-2000. (Vol. 2 Consumo de alimentos, bebidas y tabaco en unidades físicas). Madrid: I.N.E.

Isildak, O., Turkekul, I., Elmastas, M., \& Tuzen, M. (2004). Analysis of heavy metals in some wild-grow edible mushrooms from the middle black sea region, Turkey. Food Chemistry, 86, 547-552.

Jonnalagadda, S.B., Pienaar, D.H., \& Haripersad, K. (2006). Elemental distribution in selected Agaricus and Rhizina mushrooms in South Africa. Journal of Environmental Science and Health, Part A, 41, 507-514.
Jorhem, L., \& Sundström, B. (1995). Levels of some trace elements in edible fungi. Zeitschrift für Lebensmittel Untersuchung Forschung, 201, 311-316.

Letavayová, L., Vlčkvá, V., \& Brozmanová, J. (2006). Selenium: From cancer prevention to DNA damage. Toxicology, 227, 1-14.

Melgar, M.J., Alonso, J., Pérez-López, M., \& García, M.A. (1998). Influence of some factors in toxicity and accumulation of cadmium from edible wild macrofungi in NW Spain. Journal of Environmental Science and Health, Part B, 33(4), 439-455.

OMS. (2003). Guidelines for drinking-water quality (Vol. 2). Genève: World Health Organization.

Piepponen, S., Liukkonen-Lilja, H., \& Kuusi, T. (1983). The seleium content of edible mushrooms in Finland. Zeitschrift für Lebensmittel Untersuchung Forschung, 177, 257-260.

Quinche, J.P. (1987). Les teneurs en huit éléments traces de Lepista nuda. Mycologia Helvetica, 2(2), 173-181.

Shils, M.E., Olson, M., Shike, M., \& Ross, A.C. (2002). Nutrición en salud y enfermedad. México D.F.: McGraw Hill Interamericana.

Slejkovec, Z., van Eltern, J.T., Woroniecka, U.D., Kroon, K.J., Falnoga, I., \& Byrne, A.R. (2000). Preliminary study on the determination of selenium compounds income selenium-accumulating mushrooms. Biological Trace Element Research, 75(1-3), 139-155.

Stijve, T. (1977). Selenium content of mushrooms. Zeitschrift für Lebensmittel Untersuchung Forschung, 164, 201-203.

Whanger, P.D. (2002). Selenocompounds in plants and animals and their biological significance. Journal of the American College of Nutrition, 21, 223-252.

Whanger, P.D. (2004). Selenium and its relationship to cancer: An update. British Journal of Nutrition, 91, 1-28.

Yamac, M., Yildiz, D., Sarikurkcu, C., Celikkollu, M., \& Solak, M.H. (2007). Heavy metals in some edible mushrooms from the central Anatolia, Turkey. Food Chemistry, 103, 263-267.

Zaichick, V. (2002). Instrumental neutron activation analysis of minor and trace elements in foods in the Russian region that suffered from the Chernobyl disaster. Food and Nutrition Bulletin, 23, 191-194. 